\title{
Electromagnetic Zero Point Field as Active Energy Source in the Intergalactic Medium
}

\author{
Alfonso Rueda and Hiroki Sunahata \\ California State University, Long Beach, CA 90840 \\ E-mail: arueda@csulb.edu \\ Bernhard Haisch \\ Solar \& Astrophysics Laboratory, Lockheed Martin \\ 3251 Hanover St., Palo Alto, CA 94304 \\ E-mail: haisch@starspot.com \\ Revised version of invited presentation at \\ 35th AIAA/ASME/SAE/ASEE Joint Propulsion Conference and Exhibit \\ 20-24 June 1999, Los Angeles, CA \\ AIAA paper $99-2145$
}

\begin{abstract}
For over twenty years the possibility that the electromagnetic zero point field (ZPF) may actively accelerate electromagnetically interacting particles in regions of extremely low particle density (as those extant in intergalactic space (IGS) with $n \leq 1$ particle $\mathrm{m}^{-3}$ ) has been studied and analyzed. This energizing phenomenon has been one of the few contenders for acceleration of cosmic rays (CR), particularly at ultrahigh energies. The recent finding by the AGASA collaboration (Phys. Rev. Lett., 81, 1163, 1998) that the CR energy spectrum does not display any signs of the Greisen-Zatsepin-Kuzmin cut-off (that should be present if these CR particles were indeed generated in localized ultrahigh energies CR sources, as e.g., quasars and other highly active galactic nuclei), may indicate the need for an acceleration mechanism that is distributed throughout IGS as is the case with the ZPF. Other unexplained phenomena that receive an explanation from this mechanism are the generation of X-ray and gamma-ray backgrounds and the existence of Cosmic Voids. However recently, a statistical mechanics kind of challenge to the classical (not the quantum) version of the zero-point acceleration mechanism has been posed (de la Peña and Cetto, The Quantum Dice, 1996). Here we briefly examine the consequences of this challenge and a prospective resolution.
\end{abstract}

\section{INTRODUCTION}

The idea that the vacuum may play a fundamental role in the early development and future evolution of the universe has been "in the air" for a long time [1]. Both the Steady State and the old (pre-inflation model) Big Bang cosmological scenarios needed to invoke this idea in one way or another [2]. The Steady State proposed the perennial creation of particles (protons and electrons, presumably from the Dirac vacuum of particle-antiparticle pairs) throughout the universe. The old Big Bang model postulated a localized and instantaneous generation of all the matter and energy of the universe together with the subsequent gradual generation of space-time which sprang from literally nothing. And the new Big Bang with its more sophisticated Inflationary Cosmological Model started from the postulation of an unstable vacuum (the false vacuum) that collapsed, giving rise to the simultaneous creation of matter-energy and space-time followed by an inflation which produces a rapid expansion of space-time [3, 4, 5]. This inflation [5] was brief but extremely dynamic, and, as new space was actually being created, the process gave the illusion of being superluminal. Once this stage terminated, the universe entered its much slower current Hubble expansion $[1,5,6]$, the era in which we now are and have been for the last $12-20 \times 10^{9}$ years or so.

Copyright (c) 1999 by the American Institute of Aeronautics and Astronautics, Inc. All rights reserved. 
If the vacuum played such an active role in the initial stages of the universe, a valid question would be whether it is still playing some active role in the present expansion of the universe. Recent observational findings by two different teams of astronomers point in this direction [7,8]. It has been discovered that instead of a decreasing rate of expansion by cosmological gravitational attraction, as was assumed for many years, the universe actually experiences an accelerated rate of expansion. This accelerated rate of expansion may be physically explainable in terms of an ongoing involvement of the vacuum in energizing the expansion.

\section{ACCELERATED EXPANSION AND COSMIC VOIDS}

There is a paucity of known mechanisms by which the vacuum might yield some of its energy to steadily contribute to an accelerated expansion of the universe. Recently however within a different astrophysical context a mechanism that could accomplish this was proposed $[9,10,11]$. Rueda, Haisch and Cole [11] investigated a mechanism that could account for the phenomenon of Cosmic Voids [12]. It is now known that the structure of the universe presents a peculiar distribution of matter in which clusters and superclusters of galaxies are found in "sheets" that surround "voids", i.e., large regions of space of typical diameters around 100 Megaparsecs, which are practically devoid of matter and where no galaxies are found [1]. The whole structure may be represented by a soap-foam-like model where the particles and magnetic fields are mainly found on the sheets along with galactic clusters and superclusters, surrounding enormous spaces in between in which much lower particle densities and much smaller concomitant magnetic field intensities prevail.

It has been proposed $[10,11]$ that the ZPF of traditional quantum electrodynamics (QED), via a wellknown mechanism (discussed below), is responsible for the effect. This mechanism seems also to be involved in contributing to other astrophysical phenomena: the X-ray and gamma-ray backgrounds [13] and the acceleration of cosmic rays (CR), particularly at very high energies, $E \geq 10^{17} \mathrm{eV}$ (see [9] for an extensive review). It is important to emphasize that when the ZPF is applied as an energizing entity to produce the expansion associated with the Cosmic Voids, automatically an expansion of the universe itself must necessarily be produced.

The ZPF acceleration mechanism expands the Voids by creating a pressure imbalance, transferring energy to, and thereby increasing the pressure in, those regions where matter densities happen to be comparatively lower in the IGS plasma $[10,11]$. As a consequence the low-density regions tend to expand and to expel the trapped magnetic fields. It is well known in astrophysics that space plasma regions of higher densities are colder, while regions of lower density are almost exclusively occupied by a highly energetic (hot) plasma of electrons and ionized nuclei, mainly protons [15]. This temperature-density anticorrelation is what occurs in the Voids and it is also what is happening in many places throughout IGS. Moreover, such a distribution would be a natural outcome of the ZPF acceleration mechanism when combined with ordinary radiative collisional cooling [9].

\section{THE ZERO-POINT FIELD ACCELERATION MECHANISM}

The origins of this mechanism go back to the early work of Einstein and his immediate collaborators [16, 17]. Einstein realized that when a gas of electromagnetically interacting particles ${ }^{a}$ is submitted to the action of a random electromagnetic background (e.g., the case of thermal radiation) two simultaneous phenomena take place. Due to the action of the random electromagnetic medium, electromagnetically interacting particles become energized, steadily increasing their translational kinetic energy. They perform a random walk in velocity space that takes them, on average, systematically away from the origin. Simultaneously however, as their velocities increase the particles find themselves submerged in a random electromagnetic medium that is viewed by each of them as Doppler-shifted and thereby has lost its isotropy. This causes the random electromagnetic background to appear distorted and thereby to produce a drag force on the particle that is of a frictional character because it is exactly proportional to the velocity $\vec{v}$,

${ }^{a}$ Einstein restricted his considertion to polarizable particles. However it can be shown that in the case of monopolar particles the mechanism is even more effective $[9,18]$ 


$$
\vec{F}=-A\left[\rho(\omega, T)-\frac{1}{3} \omega \frac{\partial \rho(\omega, T)}{\partial \omega}\right] \frac{\vec{v}}{c}
$$

where $A$ is a positive constant. In a Hohlraum, at equilibrium temperature $T, \rho(\omega, T)$ represents the volumetric spectral energy density of the radiation:

$$
\rho(\omega, T) d \omega=\frac{\hbar \omega^{3}}{2 \pi^{2} c^{3}} \operatorname{Coth}\left(\frac{\hbar \omega}{2 k T}\right) d \omega=\frac{\hbar \omega^{3}}{2 \pi^{2} c^{3}}\left[\frac{1}{\exp (\hbar \omega / k T)-1}+\frac{1}{2}\right] d \omega .
$$

The first term in the last parenthesis represents the thermal part: a Planck distribution at temperature $T$. It disappears at zero temperature $(T \rightarrow 0)$, leaving the last part,

$$
\rho(\omega, 0) d \omega=\rho_{0}(\omega) d \omega=\frac{\hbar \omega^{3}}{2 \pi^{2} c^{3}} d \omega
$$

This is the spectral energy density of the ZPF. It originates in quantum theory from the harmonic oscillator behavior of the individual cavity modes. At $T=0$, each individual cavity mode behaves as a quantized harmonic oscillator with minimum, or zero-point, ground state energy $\hbar \omega / 2$. When the energy in each oscillator is multiplied by the density of modes per unit volume $\left(\omega^{2} / \pi^{2} c^{3}\right)$, one obtains the ZPF spectral energy density above.

It can be shown that such ZPF is also present in free space, and cogent arguments can be given for its reality $[19,20]$. Observe however, that when the temperature is set to zero (or close to zero) $\rho(\omega, T) d \omega \rightarrow$ $\rho_{0}(\omega) d \omega$ and because of the $\omega^{3}$ dependence, the Einstein-Hopf drag disappears. This last fact, first realized by Boyer [21], is at the basis of the ZPF acceleration mechanism. So, under circumstances in which there are negligible particle collisions and negligible ambient radiation fields other than the the ZPF, when the temperature is low or negligible, particles are still translationally energized by the random background $\mathrm{ZPF}$ radiation. But the Einstein-Hopf drag due to the ZPF is zero. In the original formulation of a ZPF acceleration mechanism $[21,16]$, it was assumed for simplicity that the translational displacement of the particle was restricted to a single dimension (say, the $x$-axis) and that the internal dipole vibrated along a single direction (say the $z$-axis). These restrictions were removed by one of us when proposing this concept as a mechanism for the actual acceleration of cosmic ray (CR) particles in IGS $[14,9]$. Soon after it was realized [18] that monopolar particles could also be accelerated by the ZPF, but in a much more effective manner than polarizable particles. This conforms with the well-known observational constraint on CR acceleration mechanisms that restricts the acceleration to fully ionized nuclei [9]. Another well-known constraint is that electrons appear in CR only at the very low energies, $E \leq 10^{12} \mathrm{eV}$, and not beyond. This could be explained [22] by the ultrarelativistic Zitterbewegung that, because of a time dilation effect, decorrelates the actions of the electric and of the magnetic fields in the Einstein-Hopf mechanism. This therefore prevents the acceleration of electrons to ultrahigh CR energies. This decorrelation does not take place in the case of the much more massive and sturdy protons that, if allowed, can be carried up to the highest CR energies beyond $10^{20} \mathrm{eV}[9]$.

The ZPF CR acceleration mechanism can be derived in a quantum way [23, 24]. It was found that it occurs in a time-symmetric or Wheeler-Feynman version of QED. But acceleration does not occur in the more ordinary time-unidirectional version of QED [24, 9]. However, as the time-symmetric QED version and the time-unidirectional version are equivalent (as long as certain initial boundary conditions are assumed for the radiation in space-time and as those conditions seem to hold in the original universe [25]) there is no clear reason for taking one or the other versions of QED: no reason other than the fact that we are more used to the time-unidirectional version.

It is moreover very interesting to mention that both the Wheeler-Feynman version of QED and the classical stochastic theory give exactly the same final form for the translational kinetic energy rate of growth $\Omega$, namely 


$$
\Omega=\frac{3}{5 \pi}\left(\Gamma \omega_{0}\right)^{2}\left(\frac{\hbar \omega_{0}}{m c^{2}}\right)\left(\hbar \omega_{0}\right) \omega_{0}
$$

where $\Gamma$ is the Abraham-Lorentz parameter $\left(\Gamma=2 e^{2} / 3 m c^{3}\right)$, with $e$ the charge and $m$ the mass of the particle (proton). The frequency $\omega_{0}$ is a parameter that depends on other considerations, e.g., what entity really performs the oscillations, whether the whole proton or some component (like quarks, proton vibration modes, etc.) inside the proton. In the most simplistic case $\omega_{0}$ comes to be half the proton Compton frequency $\left(\omega_{0}=m c^{2} / 2 \hbar\right)$, but this applies only under unrealistic strictly subrelativistic considerations. In practice $\omega_{0}$ is taken as a free-parameter to be phenomenologically fitted by observation.

The ZPF acceleration mechanism could be found to satisfy all standard CR observational constraints [26, 9], certainly at energies $E \geq 10^{17} \mathrm{eV}$. But lower energies could not be immediately excluded, though the situation there was somewhat less certain [9]. The least that we can say then is that the mechanism, up to now, seems to be one of the strongest contenders for CR acceleration at ultrahigh $E \geq 10^{17} \mathrm{eV}$ energies.

\section{CHALLENGE TO THE ZPF ACCELERATION CONCEPT}

Recently in their 1996 textbook on the theory of Stochastic Electrodynamics (SED) and related theories, de la Peña and Cetto [20] have challenged at least some aspects of the ZPF acceleration concept. In a lucid reanalysis [27] of the Boyer derivation [21] of the translational kinetic energy growth, they argue that if an arguably more realistic non-Markovian stochastic process is assumed for the phenomenon in its classical form, no systematic translational kinetic energy growth takes place. This would then rather fit, according to de la Peña and Cetto, the time-unidirectional version of the QED acceleration mechanism that indeed yields no systematic translational kinetic energy growth [24, 9]. We discuss the de la Peña and Cetto argument in the Appendix.

Formally, of course, and once the non-Markovian behavior is assumed, the argument of de la Pẽna and Cetto seems faultless. The situation however is far from clear. Time-symmetric QED [25] still gives the acceleration and there is no certainty at all that even when a classical viewpoint is implemented, the process has to be non-Markovian, i.e., having some memory. Moreover, recent work of Cole [28] strongly suggests the thermodynamics soundness of the ZPF acceleration mechanism both in its physical and in its astrophysical context. This step is important. Before this there remained a thermodynamic challenge to ZPF acceleration [29]. Apparently, ZPF acceleration seemed to violate standard interpretations of the first and of the second laws of thermodynamics. It has been shown that this is indeed not the case [28].

\section{DISCUSSION}

Given the overwhelming astrophysical explanatory possibilities of the ZPF acceleration mechanism to mention a few, accelerated cosmic expansion, ultrahigh energies CR, part of the X-ray and the gamma-ray backgrounds, Cosmic Voids, etc. - it is of paramount importance to clarify the situation and decide if the de la Peña and Cetto challenge is or is not a surmountable difficulty.

There are also other important possibilities for the ZPF acceleration mechanism. If valid, the mechanism should eventually provide a means to transfer energy, back and forth, but most importantly forth [9], from the vacuum electromagnetic ZPF into a suitable experimental apparatus. A more far-fetched but not trifling possibility is that a better understanding of the Einstein-Hopf process, that accompanies ZPF acceleration, would lead to an understanding of the recently proposed ZPF contribution to inertia [30], also presumably to some means for influencing inertia, and by the Einstein Principle of Equivalence, also gravity. This has very interesting prospective engineering applications.

\section{ACKNOWLEDGEMENTS}

$\mathrm{BH}$ and AR acknowledge partial support from NASA research contract NASW-5050. AR and BH acknowledge interesting exchanges with Prof. Daniel C. Cole (Boston University). 


\section{APPENDIX: Possible suppression of the secular ZPF acceleration}

De la Peña and Cetto [20] have reached the conclusion that the zero-point field may not produce secular acceleration. In this Appendix, the arguments leading to this conclusion are discussed.

\section{Dipole Oscillator Model}

The Boyer SED approach to the problem [21] is based upon the model originally developed by Einstein and Hopf $[16,17]$. In this model, that for simplicity we follow here, it is assumed that the internal particle motion is in the $x$-direction and the oscillator dipole vibrates along the $z$-axis. (An extension to fully three-dimensional motions was obtained in [14].)

During a time interval $\delta t$, this oscillator experiences two forces due to electromagnetic radiation, namely the impulse $\Delta$ transferred to the dipole via the interaction with the fluctuating field, and the force of resistance $R p$ due to the anisotropy of the field as seen by the moving particle. Then, if at time $t$, the momentum of the translational motion of the oscillator is $p$, after a short time $\delta t$, its momentum becomes $p+\Delta-R p \delta t$. Since in equilibrium the mean-square momentum has to be constant in time, we get the equilibrium condition,

$$
\left\langle p^{2}\right\rangle=\left\langle(p+\Delta-R p \delta t)^{2}\right\rangle,
$$

where the impulse $\Delta=\int F d t$, and the drag coefficient $R$ are given respectively by

$$
\left\langle\Delta^{2}\right\rangle=\left\langle\left(\int_{t}^{t+\delta t} d t e z \frac{\partial E_{z}}{\partial x}\right)^{2}\right\rangle=\frac{4 \tau \pi^{4} c^{4}}{5 \omega^{2}} \rho^{2}(\omega, T) \delta t
$$

and

$$
R=\frac{6 \pi^{2} c \tau}{5 m}\left(\rho-\frac{1}{3} \omega \frac{\partial \rho}{\partial \omega}\right) .
$$

Equation (A1), when expanded and neglecting the term of second order in $\delta t$ yields

$$
\left\langle\Delta^{2}\right\rangle+2\langle p \Delta\rangle-2 R\left\langle p^{2}\right\rangle \delta t-2 R\langle p \Delta\rangle \delta t=0 .
$$

At $T=0$, however, there is no drag force since $\rho_{0}$ is Lorentz-invariant, so that the above equation yields

$$
\left\langle\Delta^{2}\right\rangle_{0}+2\langle p \Delta\rangle_{0}=0 .
$$

This suggests that since $\left\langle\Delta^{2}\right\rangle_{0} \neq 0$ due to the presence of the zero-point field, the momentum $p$ and the fluctuation $\Delta$ have to be correlated, contrary to the assumption $\langle p \Delta\rangle=0$ correctly made by Einstein and his coworkers, but only for the case of pure thermal radiation. It is here where de la Peña and Cetto disagree with Boyer [21] who assumed even when the ZPF is present that $\langle p \Delta\rangle=0$ always. So, de la Peña and Cetto sensibly claim that the fluctuation at a given time is not independent of past ones, i.e., the process $\delta p=p-\bar{p}$ is not Markovian and the system acquires a certain degree of memory in its interactions with the ZPF.

Now let us combine (A4) with (A5) using the approximation $\langle p \Delta\rangle \approx\langle p \Delta\rangle_{0}$ (which is justified because the thermal component of the field is not expected to contribute significantly to the correlation $\left.\langle p \Delta\rangle_{0}\right)$ to yield

$$
\left\langle\Delta^{2}\right\rangle-\left\langle\Delta^{2}\right\rangle_{0}=2 R\left\langle p^{2}\right\rangle \delta t-R\left\langle\Delta^{2}\right\rangle_{0} \delta t .
$$

It can be shown and is well-known to experts dealing with the model of Einstein and Hopf in SED that the stochastic average of the square of the fluctuating impulse $\left\langle\Delta^{2}\right\rangle_{0}$ is of order $\delta t$. Thus, the last term is of order $(\delta t)^{2}$ and can be neglected. Hence the equation simplifies to 


$$
\left\langle\Delta^{2}\right\rangle-\left\langle\Delta^{2}\right\rangle_{0}=2 R\left\langle p^{2}\right\rangle \delta t
$$

The first term includes both the thermal and zero-point fluctuations and reduces to $\left\langle\Delta^{2}\right\rangle_{0}$ at $T=0$. The drag force on the right hand side is also zero at $T=0$ due to the $\omega^{3}$ dependence of the $\rho_{0}$. Since both sides reduce to zero at $T=0$, it can be argued that $\left\langle\Delta^{2}\right\rangle_{0}$ is no longer of the form const $\times \delta t$ that was responsible for the steady translational kinetic energy growth. Hence no acceleration of a free particle due to the zero-point field. The averaged square of the fluctuating impulse should be a constant as in standard theory.

\section{Quantum analysis}

Our quantum work $[9,23,24]$ does not entirely support this De la Peña and Cetto conclusion. We find that the secular acceleration disappears only under ordinary time-unidirectional QED. However, the secular acceleration mechanism is resurrected and in full force (with a much more detailed algebraic expression that reduces in a suitable limit to the standard classical case) under the Wheeler-Feynman time-symmetric form of QED. A detailed discussion of this is found in the Appendix of [9]. Our new exploration of this subject of the secular acceleration mechanism involves among other things a reanalysis of these results and a comparison of the classical Markovian case of the traditional ZPF SED secular acceleration case, the non-Markovian counterpart of De la Peña and Cetto as well as the corresponding time-asymmetric and time-symmetric

QED versions. We intend to pursue (and have proposed in a NASA research proposal with D.C. Cole as PI) to perform an experimental approach to the problem in order to check if the secular acceleration can be validated for example in a Paul trap.

\section{REFERENCES}

[1] Börner, G. The Early Universe (Springer-Verlag, Heidelberg, 1988) and references therein.

[2] Weinberg, S. Gravitation and Cosmology (Wiley, New York, 1972) and references therein.

[3] Gliner, E.B., Sov. Phys. JETP 22, 378 (1965).

[4] Sato, K., Mon. Not. R. Astron. Soc. 195, 487 (1981).

[5] Guth, A., Phys. Rev. D 23, 347 (1981).

[6] Linde, A.D., Phys. Lett. 108B, 389 (1982)

[7] Perlmutter, S. et al, LBNL, preprint 41801 (1998) and for all relevant detailed updated information: www-supernova.lbl.gov

[8] Perlmutter, S. et al., Nature 391, 51 (1998) and references therein.

[9] Rueda, A., Space Science Reviews 53, 223-345 (1990) and references therein.

[10] Rueda, A., Phys. Lett. A 147, 423 (1990).

[11] Rueda, A., Haisch, B. \& Cole, D.C. Astrophys. J. 445, 7 (1995).

[12] De Lapparent, V., Geller, M.J. \& Huchra, J.P., Astrophys. J. 302, L1 (1986), and references therein.

[13] Rueda, A., in The Galactic and Extragalactic Background Radiation, IAU Symposium 139, Heidelberg, S. Bowyer and C. Leinert, eds., (Kluwer, Dordrecht, 1990) pp 424-425.

[14] Rueda, A., Nuovo Cimento A 48, 155 (1978).

[15] Ostriker, J., in Arons, J., McKee, C. \& Max, C., (eds.), Particle Acceleration Mechanisms in Astrophysics (AIP, New York, 1979) p. 357.

[16] Einstein, A. \& Hopf, L., Ann. Phys. (Leipzig) 33, 1105 and 1096 (1910).

[17] Einstein, A. \& Stern, O., Ann. Phys. (Leipzig) 40, 551 (1913).

[18] Rueda, A. Phys. Rev A 23, 2020 (1981). 
[19] Brody, T., The Philosphy Behind Physics (Springer Verlag, Heidelberg 1993) L. de la Peña \& P.E. Hodgson (eds.).

[20] de la Peña, L. \& Cetto, A.M. The Quantum Dice (Kluwer, Dordrecht, 1996).

[21] Boyer, T. H., Phys. Rev. 182, 1374 (1969).

[22] Rueda, A. and Cavalleri, G., Nuovo Cimento C 6, 239 (1983).

[23] Rueda, A., Phys. Rev. A 30, 2221 (1984).

[24] Rueda, A. Nuovo Cimento B 96, 64 (1986).

[25] Davies, P.C.W. The Physics of Time Asymmetry (Univ. Calif. Press, Berkeley, 1974) and refernces therein.

[26] Rueda, A. Nuovo Cimento C 6, 523 (1983).

[27] Ref. [20] pp. 147-152 and pp. 252-253.

[28] Cole, D.C. Phys. Rev. E 51, 1663 (1995)

[29] Ref [9], p. $299 \mathrm{fff}$.

[30] Rueda, A. \& Haisch, B.,Found. Phys., 28, 1057 (1998); also Physics Letters A, 240, 115 (1998). 